\title{
Non-Cutaneous Leiomyosarcoma
}

National Cancer Institute

\section{Source}

National Cancer Institute. Non-Cutaneous Leiomyosarcoma. NCI Thesaurus. Code C121583.

A leiomyosarcoma arising from an anatomic site other than skin. 